\title{
Pathology of primary central nervous system lymphoma
}

\author{
Deborah L. Commins, M.D., Ph.D. \\ Department of Pathology, University of Southern California Keck School of Medicine, \\ Los Angeles, California
}

\begin{abstract}
$\checkmark$ The understanding of primary central nervous system lymphoma (PCNSL) has lagged behind that of the much more common systemic, nodal lymphomas. Reasons for this include the relative rarity of PCNSL and the fact that these lesions differ in some ways from their histologically similar systemic counterparts. The purpose of this paper is twofold: first, the author provides concise descriptions of the pathological features of both common and uncommon types of PCNSL while discussing issues such as the confusion surrounding histological classification of PCNSLs, problems related to intraoperative diagnosis, and the appropriate diagnostic work up. Second, the author discusses a small number of molecular studies to demonstrate the great promise they offer. Such studies have already clarified some issues, including the category of lymphocyte from which the majority of PCNSLs are derived. Hopefully in the future these studies will help guide treatment decisions.
\end{abstract}

\section{KEY WoRdS • central nervous system • lymphoma • stereotactic biopsy • Epstein-Barr virus}

$\mathrm{P}$ RIMARY central nervous system lymphoma is defined as an extranodal non-Hodgkin lymphoma located in the craniospinal axis without evidence of a systemic primary tumor. It usually presents as an intracerebral mass but may occur initially in the orbit, leptomeninges, or spinal cord. Overall, at the time of diagnosis, the leptomeninges are involved in approximately $40 \%$ of cases and the eyes in approximately $20 \% .{ }^{30}$ The spinal cord is involved in $1 \%$ of cases, and systemic metastases are found in approximately $5 \%$.

Although the incidence of PCNSL has increased dramatically in recent years, it is still a rare tumor, much less common than nodal lymphoma. ${ }^{37}$ Primary CNS lymphoma accounts for approximately 3 to $4 \%$ of primary brain tumors. These highly aggressive lesions leave patients with a median survival time of only a few months when left untreated or when treated with surgery alone..$^{25}$ Although the use of radiation and/or chemotherapy improves survival, some treatment regimens are associated with a high incidence of neurotoxicity, especially in patients older than 60 years of age. ${ }^{2}$

In this article the pathological features of PCNSL are described, including the gross and microscopic appearance, as well as the role of stereotactic biopsy in diagnosis and

\footnotetext{
Abbreviations used in this paper: ALCL $=$ anaplastic large-cell lymphoma; CNS = central nervous system; DLBCL = diffuse large B-cell lymphoma; $\mathrm{EBV}=$ Epstein-Barr virus; $\mathrm{GFAP}=$ glial fibrillary acidic protein; HAART $=$ highly active antiretroviral treatment; MZBCL = marginal zone B-cell lymphoma; PCNSL = primary CNS lymphoma; PIOL = primary intraocular lymphoma; REAL $=$ Revised European-American Classification of Lymphoid Neoplasms; WHO = World Health Organization.
}

management. Both the common (or "typical") and the uncommon forms of PCNSL will be discussed. The latter include low-grade and T-cell tumors, lymphomatosis cerebri, and dura-based lymphomas. Selected molecular studies addressing issues such as the derivation of PCNSL will be included. Epstein-Barr virus-related PCNSLs arising in the setting of immunodeficiency will be discussed separately because they differ in some respects from PCNSL seen in immunocompetent individuals. As a result, many recent studies are restricted to either immunocompetent or immunocompromised populations.

\section{Overview of Classification of PCNSLs}

Histologically, PCNSLs resemble systemic lymphomas, although a greater proportion of PCNSLs are highgrade and more than $95 \%$ are B-cell lymphomas..$^{30}$ The intraparenchymal cases, which represent the large majority of PCNSLs, are nearly always diffuse in architecture. Beyond these very general statements, any discussion of the classification of PCNSLs becomes complicated by a number of factors. At least three different classification systems (the Kiel, the Working Formulation, and the REAL) have been applied to PCNSL. Nevertheless, none of these classification systems, which were developed on the basis of studies of the nodal lymphomas, deals specifically with PCNSL.

According to the Kiel classification, common subtypes include immunoblastic, centroblastic, lymphoblastic, and low-grade lymphomas. Many PCNSLs are unclassifiable by this system, however, and there is large variation from study to study in the relative frequencies of the various subtypes. ${ }^{29}$ Application of the Working Formulation to 


\section{L. Commins}

PCNSL results in the most common types being identified as DLBCLs, followed by diffuse large cell immunoblastic and diffuse small cleaved cell lesions. Interstudy variability is also high when this classification scheme is used. Given that there is no convincing evidence for any clinical significance of classifying PCNSLs according to either the Kiel or the Working Formulation, ${ }^{30}$ subtyping PCNSL in this manner is not generally emphasized in the clinical setting.

Using the REAL ${ }^{17}$ or the closely related WHO classification schemes, which address extranodal lymphomas if not specifically PCNSL, almost all are classified simply as DLBCLs. Of those that do not fit into that category, 1 to $3 \%$ in Western countries have a T-cell phenotype, ${ }^{14,25}$ and approximately $3 \%$ are low-grade lymphomas of either Bor T-cell origin. ${ }^{20}$

\section{The DLBCL: the "Typical" PCNSL}

Diffuse large B-cell tumors in immunocompetent individuals will serve as the prototype because studies of the rarer forms of PCNSL are relatively few and mainly consist of case reports. The EBV-related tumors in immunosuppressed individuals are histopathologically quite similar, and any variation from the prototype will be discussed subsequently.

\section{Gross Appearance}

The typical PCNSL seen in immunocompetent individuals presents as a supratentorial, deep-seated, generally solitary mass $(65 \%){ }^{23}$ The most common locations are the frontal lobe, basal ganglia, and corpus callosum. The lesions tend to be large $(>2 \mathrm{~cm})$ and vary in circumscription. Some are well-circumscribed masses that are pale in color, with a relatively homogeneous appearance on the cut surface. Others are so diffusely infiltrative as to be essentially indistinguishable from surrounding brain. Figure 1 shows a PCNSL for which it is evident on gross examination that the lesion is a unilateral enlargement of the basal ganglia, with only the vague impression of a mass. There may be red-brown areas of hemorrhage and/or yellow areas of necrosis, as shown in the multifocal tumor in Fig. 2.

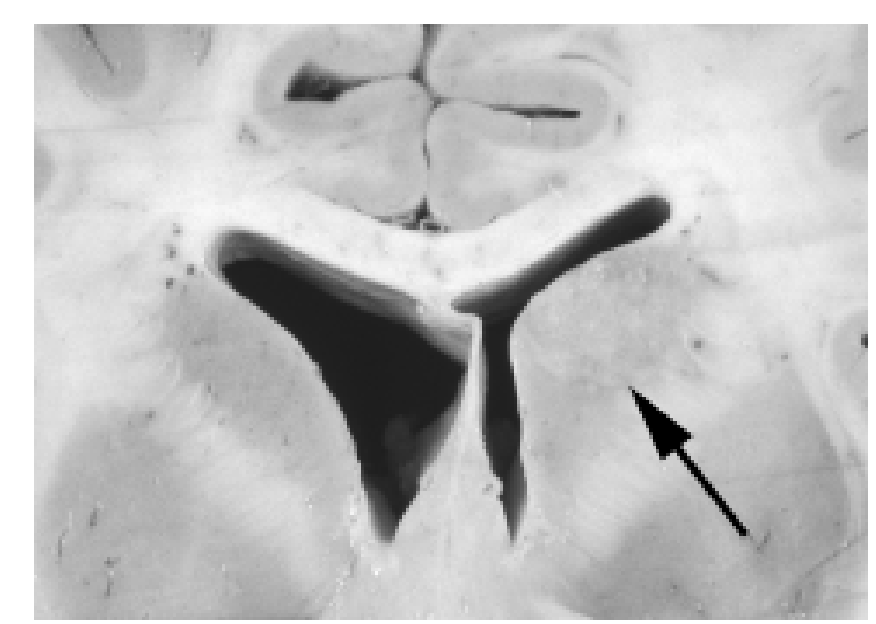

FIG. 1. Photograph of a coronal section of the brain obtained at autopsy showing enlargement of the caudate nucleus by lymphoma (arrow) without formation of a discrete mass.

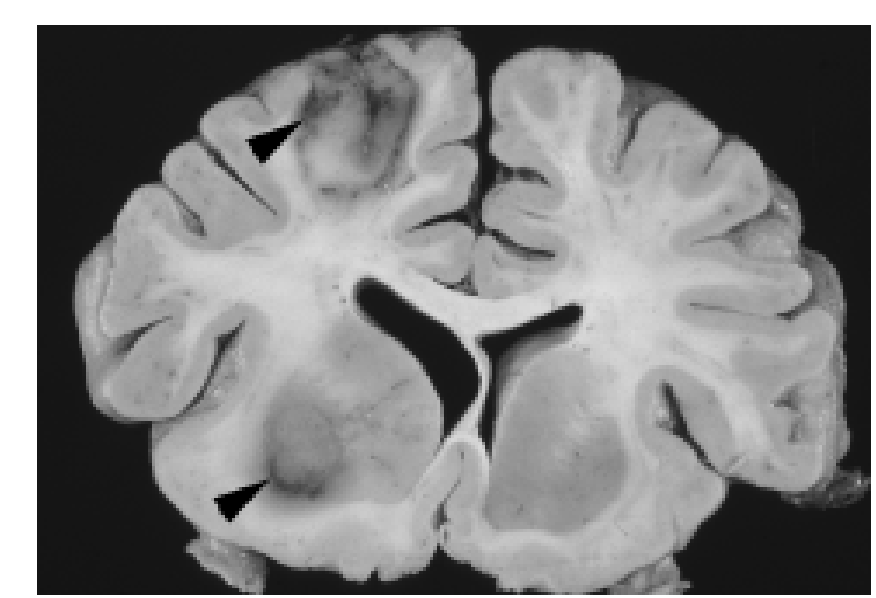

FIG. 2. Photograph of a coronal section of the brain obtained at autopsy showing multiple masses of lymphoma. The dark brown areas within the masses represent hemorrhage (arrowheads).

\section{Microscopic Appearance}

Larger Biopsy Specimens, Resections, or Autopsy Samples. Although the majority of PCNSLs are diagnosed based on stereotactic biopsy findings, the histopathological features are best appreciated in larger specimens, so these will be discussed first.

Typical PCNSLs demonstrate a striking angiocentric pattern, particularly at the edge of the mass. Blood vessels are seen, and these are surrounded by cuffs of malignant lymphocytes (Fig. 3). A special stain for reticulin often shows deposition of this material in concentric layers around the blood vessels (Fig. 4). The tumor infiltrates the brain parenchyma between blood vessels as small clusters and individual cells. Confluent areas of tumor may show necrosis, with residual viable tumor cells being found mostly around blood vessels (Fig. 5). The boundary of the tumor may be relatively discrete, but it is more common for perivascular cuffs and single infiltrating lymphoma cells to be found at some distance from the tumor mass.

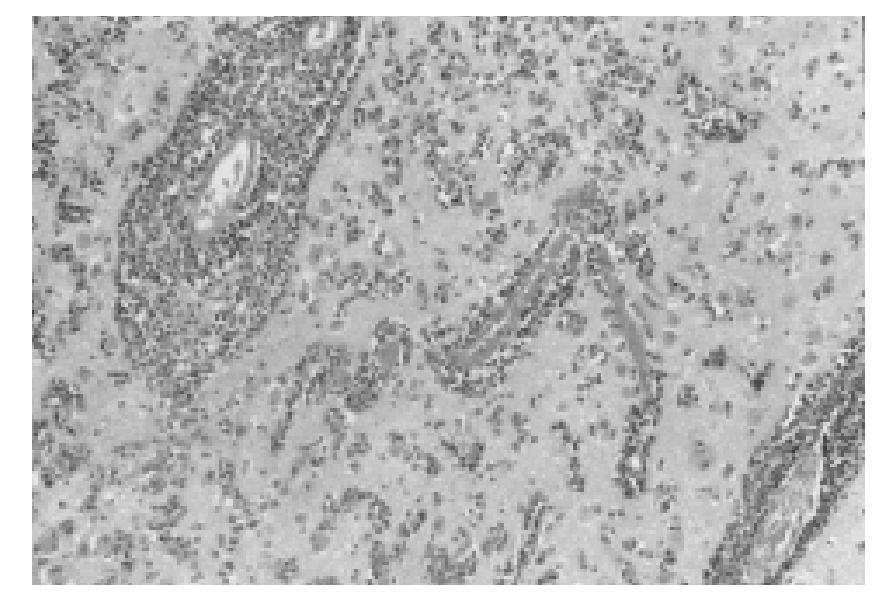

FIG. 3. Photomicrograph in which an angiocentric pattern with lymphoma cells clustering around and infiltrating blood vessels is shown that is characteristic of PCNSL. H \& E, original magnification $\times 200$. 


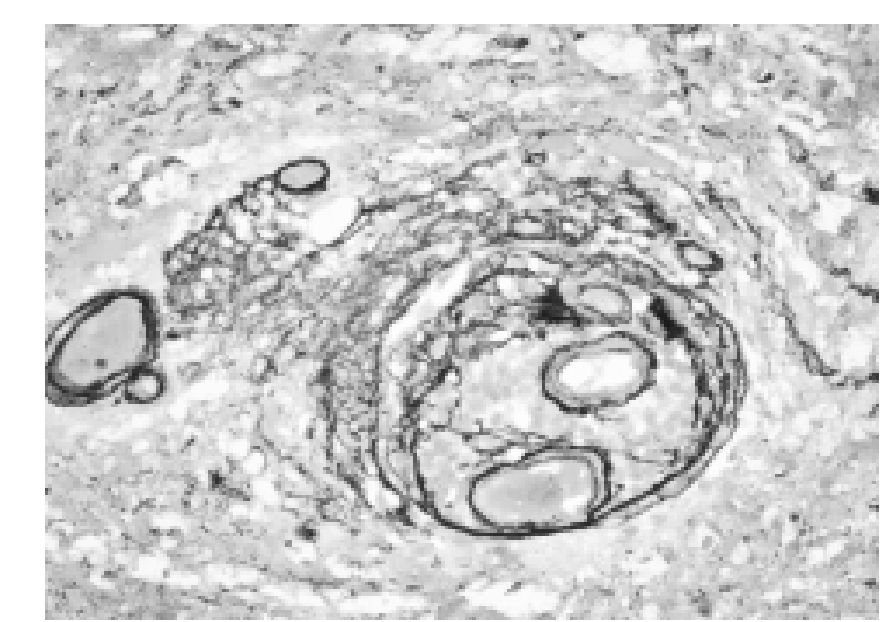

Fig. 4. Photomicrograph showing a characteristic concentric deposition of reticulin around blood vessels in PCNSL. Reticulin stain, original magnification $\times 400$.

The cells lack a cohesive appearance, without formation of glands or other structures (Fig. 6). The appearance of the nuclei ranges from round to indented or cleaved, and the nuclei have prominent nucleoli. The cells have only a small amount of basophilic cytoplasm. There are variable numbers of mitotic figures and apoptotic cells.

Stereotactic Biopsy Specimens in Untreated Patients. The majority of PCNSLs are diagnosed based on results of stereotactic brain biopsy procedures, so the sampling is limited. Biopsy specimens may reveal solid sheets of malignant lymphocytes, as shown in Fig. 7, a scant perivascular cuffing of neoplastic cells, or an almost entirely necrotic tumor.

Stereotactic Biopsy Specimens in Steroid-Treated Patients. Steroid administration has a remarkable effect on PCNSL. Over a fairly short period of time, the lymphoma "melts away," both radiographically and pathologically. ${ }^{16}$ This steroid sensitivity is of considerable therapeutic

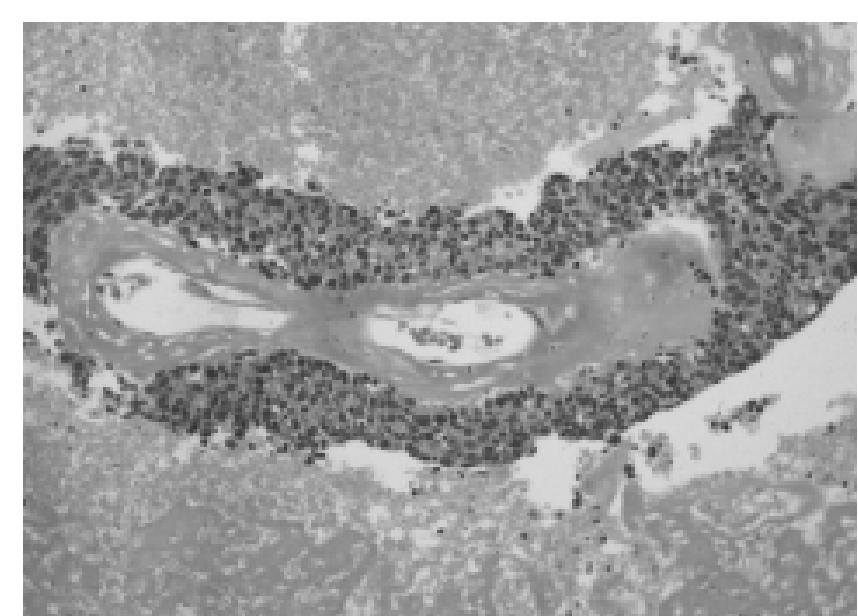

FIG. 5. Photomicrograph showing that a PCNSL may have extensive areas of necrosis. Malignant lymphocytes immediately surrounding blood vessels tend to be spared. $\mathrm{H} \& \mathrm{E}$, original magnification $\times 200$.

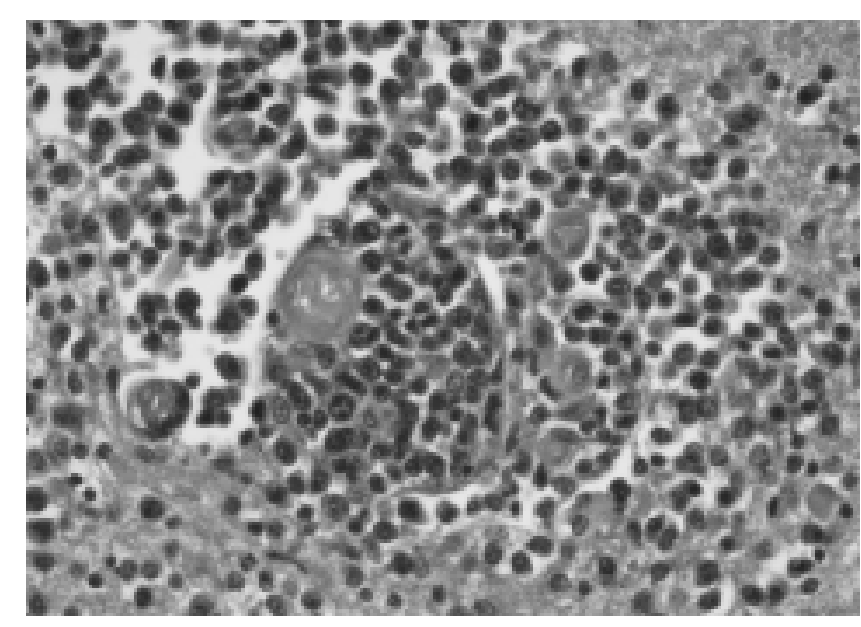

FIG. 6. Microscopic appearance of a typical PCNSL. The malignant lymphocytes are not cohesive, contain little cytoplasm, and have pleomorphic nuclei with prominent nucleoli. $\mathrm{H} \& \mathrm{E}$, original magnification $\times 400$.

value in cases in which immediate reduction of mass effect is imperative, and it may be of some diagnostic value in that it is characteristic of PCNSL. This effect is not unique to PCNSL, however, because other CNS processes, including high-grade gliomas, can sometimes respond similarly. ${ }^{43}$ Unfortunately, the response of PCNSL to steroid agents is temporary and can complicate the interpretation of subsequent specimens, making biopsy samples obtained after administration of steroid drugs less useful for the evaluation of lymphoma.

A biopsy sample obtained during the period of tumor regression after steroid administration is depicted in Fig. 8 , and it shows gliotic brain parenchyma containing an abnormal deposition of reticulin. The only lymphocytes remaining are small, nonneoplastic, $\mathrm{CD}^{+} \mathrm{T}$ cells. Although not present in this example, sheets of $\mathrm{CD}^{+} 8^{+}$macrophages can sometimes be seen.

Differential Diagnosis. The main diagnostic difficulty

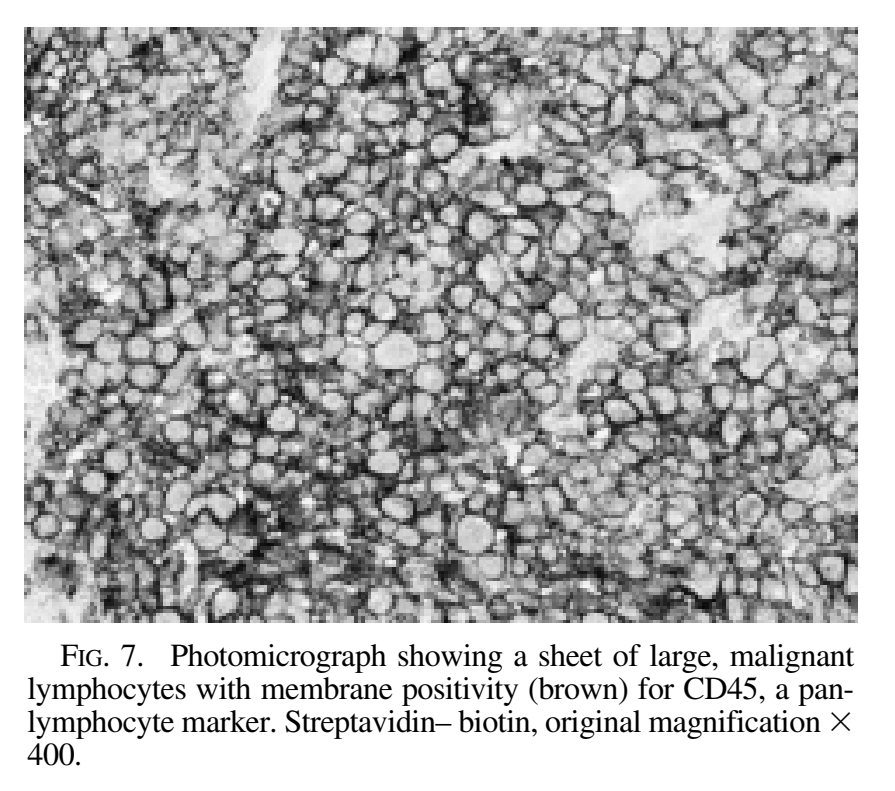




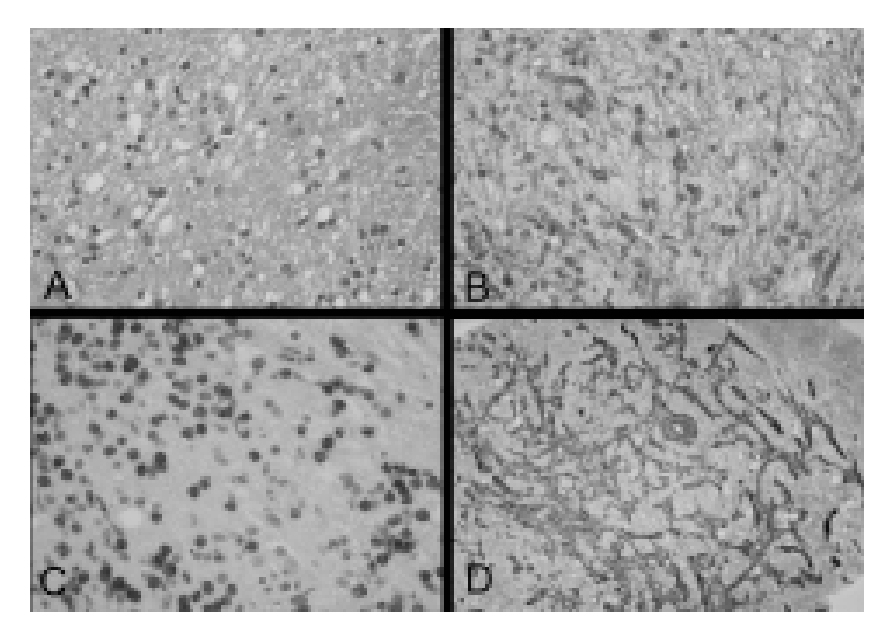

FIG. 8. Photomicrographs showing tissue sections prepared from a stereotactic biopsy specimen of a PCNSL obtained after treatment with steroid agents. A: The malignant lymphocytes have disappeared. The brain parenchyma is coarse and gliotic in appearance. $\mathrm{H} \& \mathrm{E}$, original magnification $\times 400$. B: This immunohistochemical stain for GFAP demonstrates reactive gliosis by staining some astrocytic cell bodies and numerous processes brown. Streptavidin-biotin, original magnification $\times 400$. $\mathrm{C}$ : The remaining lymphocytes are small, nonneoplastic $\mathrm{T}$ cells, as demonstrated by this positive immunohistochemical stain for the T-cell antigen CD3. Streptavidin- biotin, original magnification $\times$ 400. D: There is widespread abnormal deposition of reticulin. Reticulin stain, original magnification $\times 200$.

from a histopathological perspective is in distinguishing lymphoma from a high-grade glioma, especially when the infiltrating edge is sampled. In general, gliomas are more infiltrative and show more nuclear pleomorphism. Nevertheless, the nuclei of oligodendroglial cells can be quite uniform and, on frozen section, can be difficult to differentiate from infiltrating lymphoma cells. Other "small blue cell" tumors such as small cell carcinoma or a primitive neuroectodermal tumor can be a consideration. The clinical setting as well as cytomorphological features can be useful in differentiating these tumors from PCNSL. Both of the aforementioned tumors are more cohesive than lymphoma. In addition, small cell carcinomas generally show nuclear molding, a feature not seen in PCNSL. Any doubt can usually be resolved by the results of immunohistochemical studies on the permanent sections.

Immunohistochemical Studies. Immunohistochemical staining performed in a routine diagnostic workup includes a stain for CD45 (also known as a common leukocyte antigen, which stains both B and T cells), CD20 (a Bcell marker), and CD3 (a T-cell marker). In PCNSL, an antibody to CD20 shows staining of the large atypical lymphocytes, whereas the CD3 marker yields positive staining of the small, admixed benign $\mathrm{T}$ lymphocytes. Immunostaining for GFAP can be performed to identify astrocytes and to highlight the gliosis of the infiltrated brain parenchyma. More extensive characterization of the tumor is generally not necessary. Other immunohistochemical stains that may be useful in selected cases include immunoglobulin light chains to demonstrate monoclonality and a proliferation marker such as a MIB-1. The MIB1 marker is rarely used because the percentage of cells labeled does not seem to correlate with the prognosis.,10
The MIB-1 labeling index is generally high in PCNSL, often greater than $50 \%$.

Intraoperative Diagnosis Based on Microscopy. The angiocentric pattern typical of PCNSL is especially useful in the evaluation of frozen sections, in which artifact can severely obscure cytological features. For this reason, a smear preparation is often helpful because cytological detail is preserved. Smear preparations however, have their own difficulties. A pathologist accustomed to interpreting fine-needle aspirates of nodal lymphomas may be confused by smear preparations made from a portion of a stereotactic biopsy specimen of a PCNSL. For example, if an area of the tumor is sampled in which the lymphoma is predominantly perivascular, the smeared tissue will clump together due to gliosis and reticulin deposition, and relatively small numbers of individual cells, including benign lymphocytes and macrophages, will be present in the smear (Fig. 9). In the context of a high level of clinical suspicion, based on the presence of large, atypical lymphocytes cuffing a blood vessel on a background of reactive gliosis, a diagnosis of probable lymphoma can be made. Definitive diagnosis of malignant lymphoma requires the presence of numerous malignant lymphoid cells.

\section{Molecular Studies}

Because the different types of PCNSLs range in incidence from rare to extremely rare, and because in general only a small amount of tissue is available for study, our understanding of PCNSL lags behind that of nodal lymphomas. A number of basic questions remain unanswered. Given that the CNS has no lymphatic tissue, from what origin do PCNSLs arise? Do they arise from circulating lymphoma cells that have a special affinity for the CNS and, once there, escape destruction by the immune system? Do they arise from initially nonneoplastic precursor inflammatory processes in the CNS? Are there molecular markers that can predict prognosis? Can molecular studies explain some of the peculiar features of PCNSL, such as its angio-

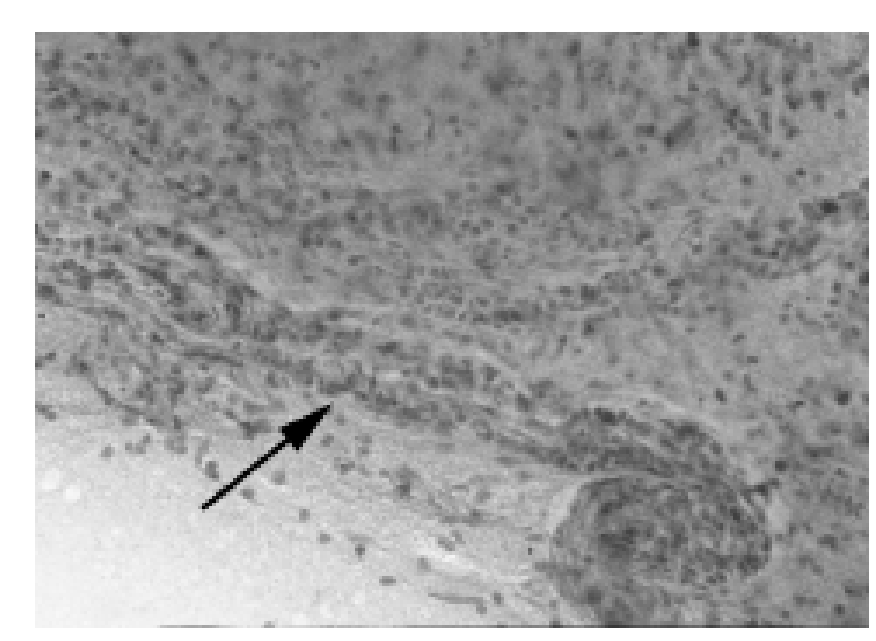

FIG. 9. Photomicrograph of a smear preparation of a stereotactic biopsy specimen of PCNSL. The tissue does not smear well because of gliosis and reticulin deposition. Malignant cells (arrow) are seen around a blood vessel but are difficult to visualize because of the thickness of the smear. Rapid H \& E, original magnification $\times 200$. 
Pathology of primary central nervous system lymphoma

centricity? In this section we review recent studies that have begun to provide some insight into these and other questions.

The Derivation of PCNSL. The Bcl-6 protein is a zincfinger transcriptional repressor required for the formation of the germinal center. ${ }^{12}$ The Bcl- 6 protein is expressed by germinal center B cells and by lesions derived from them, such as follicular lymphomas. The lymphomas derived from either naïve cells (such as mantle cell lymphoma) or postgerminal center lymphomas (for example, multiple myeloma) do not express Bcl-6.6,13

A number of studies have shown expression of Bcl-6 protein in most diffuse large B-cell PCNSLs. ${ }^{6,26,27}$ A majority $(85 \%)$ also are positive for an activation marker of late germinal center/early postgerminal center B cells (MUM1). ${ }^{6,27} \mathrm{~A}$ few expressed vs38c, a marker for plasma cells, but all were negative for CD138, another marker of plasmacytic differentiation. These findings indicate that diffuse large B-cell PCNSL probably represents a late germinal center/early postgerminal center stage of differentiation, and thus has a more differentiated cellular origin than its nodal counterpart. In these same studies, positive staining for Bcl-6 was associated with a favorable prognosis, although in other studies an association with a worse prognosis has been found. ${ }^{10}$ The fact that most PCNSLs show a germinal center or late germinal center stage of differentiation supports the suggestion that these tumors might be derived from cells that originate outside the brain, where they were exposed to a germinal center environment.

Rosenwald, et al., ${ }^{34}$ used complementary DNA microarrays to look at gene expression in nodal DLBCL to see if any pattern could be related to prognosis after therapy. They identified three subtypes of nodal DLBCL based on the pattern of gene expression: germinal center B-cell, activated B-cell, and Type 3 tumors. Of these, only the germinal center B-cell group had a favorable prognosis. Subsequently, Rubenstein, et al., ${ }^{35}$ looked at gene expression in PCNSL compared with nodal DLBCL and found that, although there was differential expression of more than 400 genes, all of the PCNSLs could be assigned to one of the three subtypes described by Rosenwald, et al. ${ }^{34}$ The proportion of tumors belonging to each of the subtypes was different, with a larger proportion of the PCNSLs being either activated B-cell or Type 3 lesions. Differences in prognosis among the three subtypes were not examined, perhaps because of the relatively small number of lymphomas analyzed. The activated B-cell type of PCNSL showed overlapping expression of germinal center genes such as $\mathrm{Bcl}-6$ (a germinal center B-cell marker) and activation genes such as cyclin D2 (an activated B-cell marker), supporting the findings in other studies, ${ }^{6,27}$ which indicated an overlapping state of differentiation in PCNSLs of the diffuse large B-cell type.

The study published by Rubenstein, et al., ${ }^{35}$ in 2006 suggests certain factors that could account for the retention of peripherally derived lymphoma cells in the CNS as well as for the angiocentric pattern displayed by PCNSLs. Interleukin-4 is a cytokine that promotes B-cell survival. Using immunohistochemistry, these investigators showed expression of interleukin-4 by endothelial cells in PCNSL, but not by endothelial cells in adjacent normal brain, in glioblastoma multiforme, or in carcinoma metastatic to the brain.

The STAT 6 factor is a signaling and transcription fac- tor that mediates the effect of interleukin-4 on gene expression. In a separate subgroup of patients, Rubenstein, et al., ${ }^{35}$ showed that increased expression of STAT 6 by a lymphoma and its endothelium was associated with tumor progression and shortened patient survival time.

\section{Pathology of Treatment-Induced Neurotoxicity in PCNSL}

Two of the most common components of treatment for PCNSL, whole-brain radiation and methotrexate chemotherapy, are neurotoxic. They act synergistically in this regard and are especially toxic when radiation treatment is administered before the chemotherapy. ${ }^{5}$ The elderly are at significantly greater risk of adverse effects than are younger patients. In one study, neurotoxicity developed in more than $80 \%$ of patients 60 years of age or older. ${ }^{1}$ This was despite the fact that they received the relatively better-tolerated regimen in which the methotrexate is given prior to radiation.

The neuropathological findings are documented in a published series of five patients with PCNSL who died severely impaired after treatment-related neurotoxicity developed. ${ }^{24}$ There was cortical atrophy with marked enlargement of the ventricles. Although none had residual tumor, all had diffuse leukoencephalopathy with myelin and axonal loss, gliosis, rarefaction, and spongiosis, and all had fibrotic thickening of the walls of the small vessels in the deep white matter. Four of the five patients had atherosclerosis in the vessels of the circle of Willis, three had cerebral infarcts, and two had areas of necrosis.

\section{Less Common Types of PCNSLs}

The T-cell Lymphomas

A finding of T-cell-derived PCNSL is rare, making it difficult to study this entity systematically. The largest series of PCNSLs with T-cell origin compiled so far includes 45 patients from 12 cancer centers in seven countries (the International Primary CNS Lymphoma Collaborative Group). ${ }^{39}$

Whereas investigators in a previous study had suggested that T-cell PCNSL might have a predilection for the posterior fossa, ${ }^{42}$ the Collaborative Group found that most T-cell PCNSLs, like most B-cell PCNSLs, are supratentorial. The original pathology reports were available for 25 cases. The disease-specific survival did not differ between those patients with lesions described as containing mainly small lymphoid cells (low grade) and those with tumors containing medium to large or anaplastic-appearing lymphoid cells (high grade). In this study no significant difference was reported in the prognosis of T-cell compared with B-cell PCNSL. In another study, T-cell PCNSLs (33\%) were more likely to be identified as low-grade lesions based on histological findings than were their Bcell counterparts (5\%), and T-cell PCNSLs with lowgrade cytological features appeared to have a better prognosis. ${ }^{42}$

\section{Anaplastic Large-Cell Lymphoma}

Approximately 20 cases of ALCL primary to the CNS have been reported in the English-language literature. ${ }^{36} \mathrm{An}$ 
ALCL is composed of cells that are generally larger than those of a DLBCL, with pleomorphic nuclei that may be single or multiple. Nucleoli are prominent and also may be multiple.

Diagnosis. Positive findings for CD30 (Ki-1) on immunohistochemical studies are crucial for the diagnosis. ${ }^{17}$ The majority of ALCLs are also positive for T-cell markers such as CD3, CD43, and CD45RO, although some are negative for both B- and T-cell markers ("null cell" tumors). Positive findings for epithelial membrane antigen, CD15, and CD45 are also variable. Earlier classification schemes included ALCL of B-cell lineage, but in the REAL and WHO classifications these are now grouped with DLBCLs. ${ }^{17}$ Thus, ALCLs are by definition negative for B-cell markers such as CD20.

Systemic ALCLs have a variety of histological appearances, including "classic," "lymphohistiocytic," and "small cell" variants. These variants have also been described in ALCL primary to the CNS. ${ }^{32}$ This morphological variability is one of the features of ALCL that can make its diagnosis challenging. The differential diagnosis includes poorly differentiated metastatic carcinoma, sarcoma, melanoma, and lymphocyte-depleted Hodgkin disease. ${ }^{17}$ Both metastatic carcinoma and ALCL may be positive for epithelial membrane antigen but, unlike metastatic carcinoma, ALCL should be positive for lymphoid markers and negative for cytokeratin. Melanoma is generally positive for S100 and/or HMB-45 and negative for lymphoid markers. Differentiation from lymphocyte-depleted Hodgkin disease depends on the results of a battery of tests for various markers. Both entities are positive for CD30 and show variable expression of CD15. Lymphocyte-depleted Hodgkin disease should be negative for epithelial membrane antigen and for T- and B-cell markers and should lack Tcell receptor and immunoglobulin gene rearrangements. ${ }^{17}$

Prognostic Factors. An ALCL may have a worse prognosis than other types of PCNSL. ${ }^{15}$ Tumor necrosis and multifocal disease may indicate a worse prognosis. Dural and leptomeningeal involvement, although common, appear to have no impact on prognosis. Although the number of tumors investigated is small, ALCL primary to the CNS, like its nodal counterpart, often shows immunopositivity with antibody to the anaplastic lymphoma kinase gene $(A L K-1) .{ }^{15}$ Immunopositivity is specific for the fusion of the $A L K$ gene on chromosome 2 with the nucleophosmin gene (NPM) on chromosome 5, which yields the fusion transcript protein $\mathrm{p} 80$. Positive findings for $A L K-1$ appear to correlate with a young patient age and a favorable prognosis. Interestingly, ALCL and mucosa-associated lymphoid tissue lymphomas are the only types of non-Hodgkin lymphoma to have a translocation that results in a fusion protein. All other translocations identified to date in non-Hodgkin lymphoma juxtapose a protooncogene to heterologous regulatory sequences. ${ }^{18}$

\section{Low-Grade PCNSLs: Pathological Features and Differential Diagnosis}

The writing group for the International Primary CNS Lymphoma Collaborative Group recently published a series of 40 low-grade PCNSLs. ${ }^{20}$ Because this was a retrospective study of cases diagnosed between 1979 and
2004 at 18 different cancer centers, the pathological findings were often not available for review and the diagnosis was obtained from old reports. This limited the ability of the Collaborative Group to classify many of the tumors according to current criteria. Thirty-two $(80 \%)$ of these low-grade tumors were B-cell lymphomas. Of these, 16 (50\%) were described as "small lymphocytic" and/or "poorly differentiated." The next largest group was lymphoplasmacytic lymphoma. The eight tumors (20\%) that were T-cell lymphomas were diagnosed based on immunohistochemical positivity for T-cell markers such as CD3 and negative results for B-cell markers such as CD20. The low-grade PCNSLs that were the subject of this study appeared similar to the usual high-grade PCNSLs in having a diffuse, angiocentric pattern.

In terms of prognosis, the Collaborative Group concluded that low-grade tumors behave in a more indolent fashion than the typical high-grade PCNSL. ${ }^{20}$ In addition, it appears that a higher percentage of low-grade tumors are T-cell lymphomas. Nevertheless, no overall difference in survival between T- and B-cell lymphomas has been demonstrated. ${ }^{39}$ Identifying subgroups of PCNSL that could be treated less aggressively is particularly important, given the significant neurotoxicity of current treatment regimens.

It can be extremely difficult to differentiate low-grade PCNSL from a reactive inflammatory process such as a viral encephalitis or multiple sclerosis. Nonneoplastic, reactive lymphocytic infiltrates in the CNS are composed predominantly of $\mathrm{T}$ cells, so a predominance of $\mathrm{B}$ cells should raise the possibility of a lymphoma. The situation may be clarified by immunohistochemical staining for kappa and lambda light chains to demonstrate monoclonality or by PCR detection of rearranged immunoglobulin heavy-chain genes..$^{20,38}$

Diagnosis of a low-grade T-cell lymphoma is more difficult. The presence of confluent sheets of T cells implicates lymphoma. If lymphoma is suspected based on either the clinical setting or histological findings, the diagnosis can be confirmed by demonstrating T-cell receptor rearrangements.

\section{Lymphomatosis Cerebri}

Lymphomatosis cerebri is a very rare condition in which PCNSL presents as a diffuse, infiltrating process without formation of a discrete mass. ${ }^{33}$ Radiographically, there is diffuse white matter involvement without enhancement. Histologically, the widespread, diffuse infiltration by lymphocytes with some perivascular cuffing can be mistaken for viral encephalitis. This problem is compounded by the presence of nonneoplastic lymphocytes, macrophages, and reactive astrocytes. The cellular composition of the infiltrate can be clarified with immunohistochemical stains for astrocytes (GFAP), macrophages (CD68), $\mathrm{T}$ cells (CD3), and B cells (CD20). The latter stain should highlight a population of enlarged, atypical cells.

\section{Primary Intraocular Lymphoma}

In contrast to systemic lymphomas, which usually involve the uveal tract, PIOLs involve the retina, vitreous, 
Pathology of primary central nervous system lymphoma

and optic nerve. ${ }^{8}$ Eighty percent of cases involve bilateral disease, and in a high percentage of patients who present with PIOL, intracerebral involvement will develop.

Almost all PIOLs are high-grade, diffuse B-cell lymphomas that are morphologically indistinguishable from those arising in the brain. Most of them stain positively for B-cell markers (CD19, CD20, and CD22) and show lightchain restriction.

These tumors may be diagnosed based on cytological examination of the vitreous showing malignant lymphoid cells with scant, basophilic cytoplasm and large, pleomorphic nuclei with prominent nucleoli. If examination of the vitreous is nondiagnostic, a chorioretinal biopsy procedure may be performed. Typical findings would include a malignant lymphoid infiltrate between the retinal pigmented epithelium and the Bruch membrane, with more widespread perivascular aggregates of tumor cells. The choroid may contain a reactive lymphocytic infiltrate. There may be hemorrhagic retinal necrosis or retinal atrophy due to detachment.

In a study comparing the pattern of immunoglobulin transcription factors in PIOL, intracerebral PCNSL, and peripheral DLBCL, investigators found that the first two had an essentially identical pattern of expression. ${ }^{11}$ The pattern of gene expression indicated that both are derived from mature B cells that have undergone the germinal center reaction. This pattern of gene expression differed in some aspects from that seen in peripheral DLBCLs, reinforcing the fact that despite their morphological similarity, PCNSLs and peripheral DLBCLs are not identical.

\section{The EBV-Related PCNSL}

Immunosuppression, congenital or acquired, is the most clear-cut risk factor for development of PCNSL. When these lesions arise in a background of immunodeficiency, they are almost always associated with EBV. This association is uncommon in immunocompetent individuals, and in this setting it is more difficult to be confident that EBV is actually playing a role in oncogenesis.

During initial infection, EBV establishes a latent infection in B lymphocytes in which only a subset of viral antigens is expressed and the infected lymphocyte is immortalized. ${ }^{19}$ Under normal circumstances, the individual's humoral and cellular immune response keeps these latently infected, activated B cells in check. Three types of EBV latency states have been identified, and Type III is seen in many EBV-related lymphoproliferative disorders that occur in immunosuppressed individuals. Type III latency is characterized by expression of all nine EBV latency proteins: Epstein-Barr nuclear antigens 1, 2, and 3A-C; LP; BARF0; and two viral membrane proteins, LMP1 and-2. Normally, cells expressing these viral antigens would be readily destroyed by EBV-specific $\mathrm{T}$ cells, so this latency pattern is only seen in the setting of immunodeficiency.

\section{Patients With AIDS}

The most common setting in which immunodeficiencyassociated PCNSL arises is in patients with AIDS. Before the advent of HAART, PCNSL was found in approximately $5 \%$ of patients with neurological symptoms, usu- ally in the late stages of AIDS. ${ }^{25}$ Since HAART has become available, most studies show a decreased incidence of PCNSL, although perhaps not to the same extent as for some opportunistic infections ${ }^{40}$ The change in incidence may depend on the characteristics of the population being studied. In one report, the incidence of PCNSL in the era of HAART was a little more than $30 \%$ of preHAART levels. ${ }^{4}$ However, the risk of developing PCNSL did not change between the two time periods among patients with comparable CD4 counts. The incidence of PCNSL remains high among the sickest patients (CD4 count $<50 / \mu \mathrm{l})$.

Compared with PCNSLs in immunocompetent individuals, in those with AIDS and in other immunosuppressed patients these lesions are more likely to be multifocal and necrotic. ${ }^{21,31}$ Histologically, PCNSLs in AIDS patients are high-grade, diffuse large B-cell tumors, often of the immunoblastic subtype. The low-grade lymphomas seen occasionally in immunocompetent individuals occur even less frequently in AIDS patients. Some lymphomas may be difficult to classify. Virtually all cases are EBV-positive.

Figure 10 shows a coronal section from the brain of an AIDS patient. The red-brown, gelatinous-appearing areas adjacent to the ventricle are typical of cytomegalovirus encephalitis. The white nodule embedded in the gelatinous area (arrowhead) is unusual, and microscopic examination (inset) shows a focus of PCNSL surrounded by megalic cells containing cytomegalovirus inclusions.

\section{Posttransplant PCNSL}

Lesions involved in systemic posttransplant lymphoproliferative disorders represent a spectrum ranging from EBV-positive, reactive, polyclonal lymphoid proliferations to overt high-grade lymphomas. ${ }^{19}$ Most of the lymphomas are EBV-positive B-cell lymphomas, although lesions causing systemic posttransplant lymphoproliferative disorders that develop later may be EBV negative.

Posttransplant lymphoproliferative disorders of the CNS have been described in case reports and small series. In contrast to lesions involved in systemic posttransplant lymphoproliferative disorders, which are heterogeneous and include low-grade proliferations, those arising in the CNS seem to be uniformly high-grade B-cell lymphomas. ${ }^{7,31}$ Similar to PCNSLs seen in patients with AIDS, these tumors tend to be multifocal, necrotic, and mostly located in the cerebral cortex and white matter and in the basal ganglia.

In one series of 12 cases, the histological features and immunophenotypes of these tumors were examined in some detail. ${ }^{7}$ Like PCNSLs arising in both AIDS patients and in immunocompetent individuals, the infiltrates were angiocentric, with invasion of blood vessel walls by tumor cells. The lesions causing posttransplant lymphoproliferative disorders of the CNS were composed of large, blastic cells with irregular nuclei, prominent nucleoli, and basophilic cytoplasm. All were monomorphic posttransplant lymphoproliferative disorders according to WHO criteria, and they were large B-cell lymphomas under the REAL classification.

Immunoperoxidase stains showed positivity of all 12 tumors for the B-cell marker CD20. Three tumors that were stained with additional B-cell markers (CD19, 


\section{L. Commins}

CD22, or CD79a) showed positivity. All the tumors that were stained for kappa and lambda light chains were lightchain restricted. Eleven were positive for EBV. The T-cell marker CD3 highlighted an admixed population of small, nonneoplastic $\mathrm{T}$ cells. Thus, the histological appearance and immunohistochemical profile are very similar to PCNSL seen in other immunodeficient states, such as AIDS.

In this small series, disease-free survival times varied from a few days to 5 years. The favorable outcome seen in some patients is probably due in part to reduction in their therapeutic immunosuppression, although they all also received chemotherapy and/or radiation treatment for their posttransplant lymphoproliferative disorder.

\section{The EBV-Associated PCNSL in Immunocompetent Patients}

These rarely arise primarily in the CNS and include some cases of Burkitt lymphoma. In a case report, Kaluza, et al., ${ }^{22}$ described an extraordinary nasal type natural killer/T-cell lymphoma arising in the frontal lobe of an otherwise healthy man. These are rare lymphomas that are most commonly of natural killer cell derivation, but are occasionally of cytotoxic T-cell origin. They are aggressive neoplasms and are one cause of "lethal midline granuloma." 9

The tumor described in this case report had a focally angiocentric pattern with vascular destruction and necrosis. The lymphoid cells were medium sized, and had a moderate amount of clear cytoplasm and pleomorphic nuclei with inconspicuous nucleoli. The tumor was positive for the natural killer cell marker CD56 and for cytoplasmic CD3 (positive in natural killer and cytotoxic T cells). It was negative for surface CD3 (positive in peripheral Tcell lymphomas) and for T-cell receptor gene rearrangements. As usual for these tumors, it was positive for EBV, specifically for EBV-encoded small nuclear RNAs.

\section{Marginal Zone B-Cell Lymphomas of the Dura Mater}

The MZBCL was first described in the mucosa-associated lymphoid tissue of the gastrointestinal tract. ${ }^{41}$ It has subsequently been divided in the WHO classification scheme into three subtypes: nodal, extranodal (mucosaassociated lymphoid tissue), and splenic, based on differences in clinical behavior. All types are believed to be derived from postgerminal center marginal zone B-cells that surround the lymphoid follicle, often referred to as monocytoid B cells because of their abundant clear cytoplasm and distinct cytoplasmic borders. They express Bcell markers such as CD20, CD19, and CD79a as well as surface immunoglobulin. They are negative for CD3, CD5, CD10, CD23, and cyclin D1 markers. ${ }^{28}$

The MZBCLs primary to the CNS, although very rare, are considered by some to be the most common low-grade PCNSL. ${ }^{41}$ They differ from the other types of PCNSL discussed so far in that they typically are dura-based and mimic meningiomas. They are also notable for their favorable prognosis and higher incidence in women. Although the dura contains no mucosa, these are considered mucosaassociated lymphoid tissue lymphomas, as are similar lesions in other tissues lacking mucosa, such as breast.

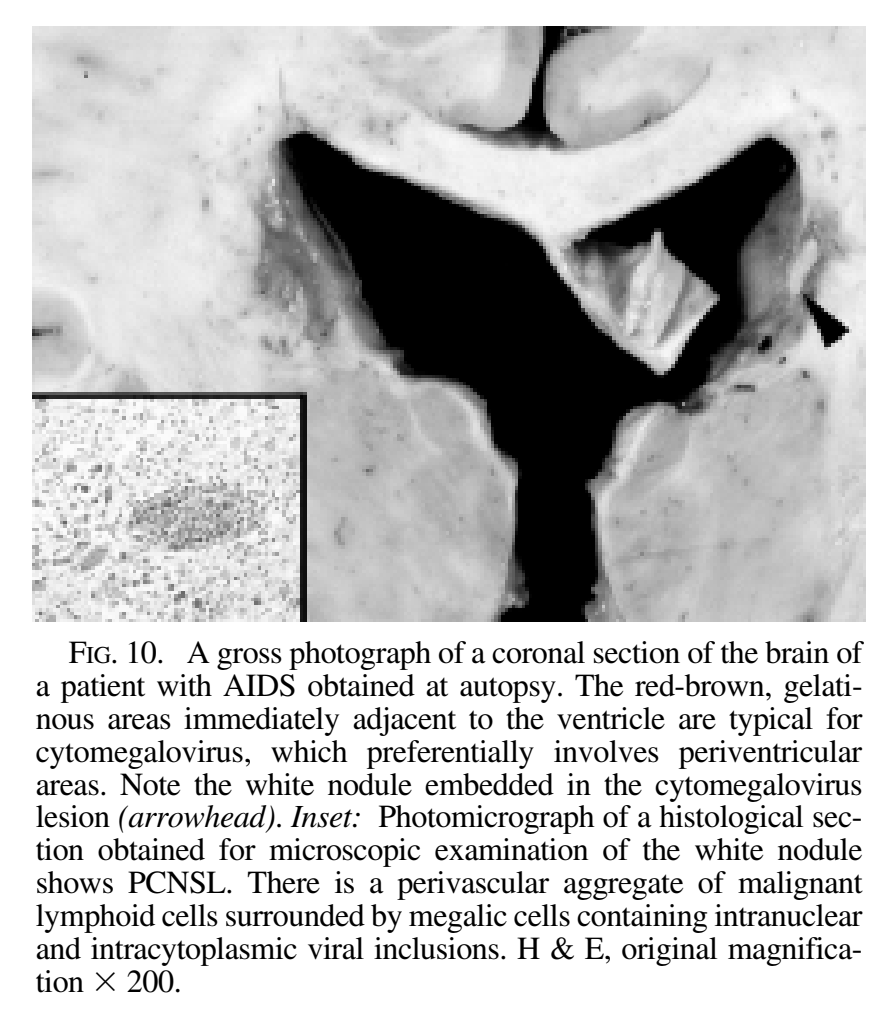

In a recent series, the histopathological and genetic findings were described in 15 cases of MZBCL primary to the CNS. ${ }^{41}$ The tumors involved mainly the dura mater and leptomeninges, with occasional instances of extension down Virchow-Robin spaces. Like MZBCLs elsewhere, the neoplastic lymphocytes were small to medium and had moderate amounts of cytoplasm and irregular nuclei, resembling follicular small-cleaved cells. Many cells were monocytoid, with distinct cytoplasmic borders and clear cytoplasm. Some tumors contained small numbers of large, immunoblast-like cells. Infiltration by plasma cells was common, and some tumors showed amyloid deposition. The pattern of immunohistochemical staining for lymphoid markers was as described earlier for MZBCLs.

Genetic abnormalities have been identified in MZBCL, the most common of which, trisomy 3 , is found in all three types (extranodal, nodal, and splenic) of this lesion. Other abnormalities found in only some subtypes of MZBCLs include $\mathrm{t}(11 ; 18)(\mathrm{q} 21 ; \mathrm{q} 21)$, resulting in the (API2-MALT1) fusion gene and immunoglobulin heavy-chain rearrangements. In MZBCLs primary to the CNS, examination for these abnormalities showed only trisomy 3 , which was present in $50 \%$ of cases. ${ }^{41}$

\section{Conclusions}

Intracerebral PCNSL is variable in its presentation. It can occur in a young, immunodeficient person in association with EBV or in an otherwise healthy older person in whom there is no association with EBV. It can be a solitary mass or multiple lesions. Occasionally it can even be diffusely infiltrative without formation of a discrete mass. A stereotactic biopsy sample may yield abnormal results but contain no neoplastic cells if there has been previous 
exposure to steroid agents. Alternatively, the biopsy sample may show sheets of malignant lymphoid cells (an easy diagnosis) or small numbers of infiltrating cells (a difficult diagnosis), or the biopsy tissue may be mostly or entirely necrotic.

Once diagnosable tumor is on the slide, however, this picture of lively variety is almost always replaced by one of monotony. Ninety-five percent of the time the tumor will be a DLBCL and only a few routine immunohistochemical stains are required to verify the diagnosis. Unfortunately, at this point there is nothing much that routine light microscopic examination can add as far as prognosis or response to treatment. Even if the PCNSL turns out to be one of the unusual types such as a T-cell lymphoma, little is certain about what difference this may make clinically. This may change as more of these very rare tumors are studied, so it is important to identify them.

Gene expression studies and other molecular approaches have already provided fascinating and potentially clinically useful information about PCNSL. As markers with important clinical significance are identified, the techniques necessary for their determination may be adapted to routine use.

\section{Acknowledgments}

I thank Roscoe Atkinson, M.D., for his invaluable assistance with the figures and Sue Ellen Martin, M.D., Ph.D., for her thoughtful editorial input.

\section{References}

1. Abrey LE, DeAngelis LM, Yahalom J: Long-term survival in primary CNS lymphoma. J Clin Oncol 16:859-863, 1998

2. Abrey LE, Yahalom J, DeAngelis LM: Treatment for primary CNS lymphoma: the next step. J Clin Oncol 18:3144-3150, 2000

3. Aho R, Haapasalo H, Alanen K, Haltia M, Paetau A, Kalimo H: Proliferative activity and DNA index do not significantly predict survival in primary central nervous system lymphoma. J Neuropathol Exp Neurol 54:826-832, 1995

4. Besson C, Goubar A, Gabarre J, Rozenbaum W, Pialoux G, Chatelet FP, et al: Changes in AIDS-related lymphoma since the era of highly active antiretroviral therapy. Blood 98: 2339-2344, 2001

5. Blay JY, Conroy T, Chevreau C, Thyss A, Quesnel N, Eghbali $\mathrm{H}$, et al: High-dose methotrexate for the treatment of primary cerebral lymphomas: analysis of survival and late neurologic toxicity in a retrospective series. J Clin Oncol 16:864-871, 1998

6. Braaten KM, Betensky RA, de Leval L, Okada Y, Hochberg $\mathrm{FH}$, Louis DN, et al: BCL-6 expression predicts improved survival in patients with primary central nervous system lymphoma. Clin Cancer Res 9:1063-1069, 2003

7. Castellano-Sanchez AA, Li S, Qian J, Lagoo A, Weir E, Brat DJ: Primary central nervous system posttransplant lymphoproliferative disorders. Am J Clin Pathol 121:246-253, 2004

8. Chan CC, Wallace DJ: Intraocular lymphoma: update on diagnosis and management. Cancer Control 11:285-295, 2004

9. Chan JKC, Jaffe ES, Ralfkiaer E: Mature T-cell and NK-cell neoplasms, in Jaffe ES, Harris NL, Stein H, Vardiman JW (eds): Pathology and Genetics of Tumours of Haematopoietic and Lymphoid Tissues. Lyon, France: IARC Press, 2001, pp 189-236

10. Chang CC, Kampalath B, Schultz C, Bunyi-Teopengco E, Logan B, Eshoa C, et al: Expression of p53, c-Myc, or Bcl-6 suggests a poor prognosis in primary central nervous system diffuse large B-cell lymphoma among immunocompetent individuals. Arch Pathol Lab Med 127:208-212, 2003

11. Coupland SE, Loddenkemper C, Smith JR, Braziel RM, Charlotte F, Anagnostopoulos I, et al: Expression of immunoglobulin transcription factors in primary intraocular lymphoma and primary central nervous system lymphoma. Invest Ophthalmol Vis Sci 46:3957-3964, 2005

12. Dent AL, Shaffer AL, Yu X, Allman D, Staudt LM: Control of inflammation, cytokine expression, and germinal center formation by BCL-6. Science 276:589-592, 1997

13. Dogan A, Bagdi E, Munson P, Isaacson PG: CD10 and BCL-6 expression in paraffin sections of normal lymphoid tissue and B-cell lymphomas. Am J Surg Pathol 24:846-852, 2000

14. Ferreri AJ, Reni M, Pasini F, Calderoni A, Tirelli U, Pivnik A, et al: A multicenter study of treatment of primary CNS lymphoma. Neurology 58:1513-1520, 2002

15. George DH, Scheithauer BW, Aker FV, Kurtin PJ, Burger PC, Cameselle-Teijeiro J, et al: Primary anaplastic large cell lymphoma of the central nervous system: prognostic effect of ALK-1 expression. Am J Surg Pathol 27:487-493, 2003

16. Geppert M, Ostertag CB, Seitz G, Kiessling M: Glucocorticoid therapy obscures the diagnosis of cerebral lymphoma. Acta Neuropathol 80:629-634, 1990

17. Harris NL, Jaffe ES, Stein H, Banks PM, Chan JK, Cleary ML, et al: A revised European-American classification of lymphoid neoplasms: a proposal from the International Lymphoma Study Group. Blood 84:1361-1392, 1994

18. Harris NL, Stein H, Coupland SE, Hummel M, Favera RD, Pasqualucci L, et al: New approaches to lymphoma diagnosis. Hematology Am Soc Hematol Educ Program 2001: 194-220, 2001

19. Heslop HE: Biology and treatment of Epstein-Barr virus-associated non-Hodgkin lymphomas. Hematology Am Soc Hematol Educ Program 2005:260-266, 2005

20. Jahnke K, Korfel A, O’Neill BP, Blay JY, Abrey LE, Martus P, et al: International study on low-grade primary central nervous system lymphoma. Ann Neurol 59:755-762, 2006

21. Johnson BA, Fram EK, Johnson PC, Jacobowitz R: The variable MR appearance of primary lymphoma of the central nervous system: comparison with histopathologic features. AJNR Am J Neuroradiol 18:563-572, 1997

22. Kaluza V, Rao DS, Said JW, de Vos S: Primary extranodal nasal-type natural killer/T-cell lymphoma of the brain: a case report. Hum Pathol 37:769-772, 2006

23. Kuker W, Nagele T, Korfel A, Heckl S, Thiel E, Bamberg M, et al: Primary central nervous system lymphomas (PCNSL): MRI features at presentation in 100 patients. J Neurooncol 72: 169-177, 2005

24. Lai R, Abrey LE, Rosenblum MK, DeAngelis LM: Treatmentinduced leukoencephalopathy in primary CNS lymphoma: a clinical and autopsy study. Neurology 62:451-456, 2004

25. Lallana E, DeAngelis LM: Lymphomas and hemopoietic neoplasms, in Berger M, Prados M (eds): Textbook of Neuro-oncology. Philadelphia: Elsevier, 2005, pp 301-309

26. Larocca LM, Capello D, Rinelli A, Nori S, Antinori A, Gloghini A, et al: The molecular and phenotypic profile of primary central nervous system lymphoma identifies distinct categories of the disease and is consistent with histogenetic derivation from germinal center-related B cells. Blood 92:1011-1019, 1998

27. Lin CH, Kuo KT, Chuang SS, Kuo SH, Chang JH, Chang KC, et al: Comparison of the expression and prognostic significance of differentiation markers between diffuse large B-cell lymphoma of central nervous system origin and peripheral nodal origin. Clin Cancer Res 12:1152-1156, 2006

28. Maes B, De Wolf-Peeters C: Marginal zone cell lymphomaan update on recent advances. Histopathology 40:117-126, 2002

29. Paulus W: Classification, pathogenesis and molecular patholo- 


\section{L. Commins}

gy of primary CNS lymphomas. J Neurooncol 43:203-208, 1999

30. Paulus W, Jellinger K, Morgello S, Deckert-Schluter M: Malignant lymphomas, in Kleihues P, Cavenee WK (eds): World Health Organization Classification of Tumours. Pathology and Genetics of Tumours of the Nervous System. Lyon, France: IARC Press, 2000, pp 198-203

31. Phan TG, O'Neill BP, Kurtin PJ: Posttransplant primary CNS lymphoma. Neuro-oncol 2:229-238, 2000

32. Ponzoni M, Terreni MR, Ciceri F, Ferreri AJM, Gerevini S, Anzalone N, et al: Primary brain CD30+ ALK1+ anaplastic large cell lymphoma ('ALKoma'): the first case with a combination of 'not common' variants. Ann Oncol 13:1827-1832, 2002

33. Rollins KE, Kleinschmidt-DeMasters BK, Corboy JR, Damek DM, Filley CM: Lymphomatosis cerebri as a cause of white matter dementia. Hum Pathol 36:282-290, 2005

34. Rosenwald A, Wright G, Chan WC, Connors JM, Campo E, Fisher RI, et al: The use of molecular profiling to predict survival after chemotherapy for diffuse large-B-cell lymphoma. $\mathbf{N}$ Engl J Med 346: 1937-1947, 2002

35. Rubenstein JL, Fridlyand J, Shen A, Aldape K, Ginzinger D, Batchelor T, et al: Gene expression and angiotropism in primary CNS lymphoma. Blood 107:3716-3723, 2006

36. Rupani A, Modi C, Desai S, Rege J: Primary anaplastic large cell lymphoma of central nervous system-a case report. J Postgrad Med 51:326-327, 2005

37. Sarkar C, Sharma MC, Deb P, Singh R, Santosh V, Shankar SK: Primary central nervous system lymphoma-a hospital based study of incidence and clinicopathological features from India (1980-2003). J Neurooncol 71:199-204, 2005

38. Schlegel U, Schmidt-Wolf IG, Deckert M: Primary CNS lymphoma: clinical presentation, pathological classification, mole- cular pathogenesis and treatment. J Neurol Sci 181:1-12, 2000

39. Shenkier TN, Blay JY, O'Neill BP, Poortmans P, Thiel E, Jahnke K, et al: Primary CNS lymphoma of T-cell origin: a descriptive analysis from the International Primary CNS Lymphoma Collaborative Group. J Clin Oncol 23:2233-2239, 2005

40. Skiest DJ, Crosby C: Survival is prolonged by highly active antiretroviral therapy in AIDS patients with primary central nervous system lymphoma. AIDS 17:1787-1793, 2003

41. Tu PH, Giannini C, Judkins AR, Schwalb JM, Burack R, O'Neill BP, et al: Clinicopathologic and genetic profile of intracranial marginal zone lymphoma: a primary low-grade CNS lymphoma that mimics meningioma. J Clin Oncol 23: 5718-5727, 2005

42. Villegas E, Villa S, Lopez-Guillermo A, Petit J, Ribalta T, Graus F: Primary central nervous system lymphoma of T-cell origin: description of two cases and review of the literature. J Neurooncol 34:157-161, 1997

43. Zaki HS, Jenkinson MD, Du Plessis DG, Smith T, Rainov NG: Vanishing contrast enhancement in malignant glioma after corticosteroid treatment. Acta Neurochir (Wien) 146:841-845, 2004

Support for this study was received from the National Neurological AIDS Bank (NNAB), Grant No. NS-38841.

Manuscript received August 16, 2006.

Accepted in final form October 4, 2006.

Address reprint requests to: Deborah L. Commins, M.D, Ph.D., Department of Pathology, University of Southern California Hospital, 1500 San Pablo Street, Los Angeles, California 90033. email: commins@usc.edu. 